\title{
BMJ Open Comparison of the efficacy and acceptability of Chinese herbal medicine in adult patients with heart failure and reduced ejection fraction: study protocol for a systematic review and network meta-analysis
}

Jing Liu, ${ }^{1}$ Jin-Jin Lu, ${ }^{2}$ Kun Zhou, ${ }^{3}$ Jie Wan, ${ }^{4}$ Yan Li, ${ }^{2}$ Xiao-Yun Cui, ${ }^{2}$ Qun Gao, ${ }^{1}$ Yan-Chao Huang, ${ }^{1}$ Si-Nai Li, ${ }^{5}$ Qiao-Zhi Dong, ${ }^{6}$ Qian Lin ${ }^{2}$

To cite: Liu J, Lu J-J, Zhou K, et al. Comparison of the efficacy and acceptability of Chinese herbal medicine in adult patients with heart failure and reduced ejection fraction: study protocol for a systematic review and network meta-analysis. BMJ Open 2018;8:e015678. doi:10.1136/ bmjopen-2016-015678

- Prepublication history and additional material for this paper are available online. To view please visit the journal (http:// dx.doi.org/10.1136/bmjopen2016-015678).

Received 28 December 2016 Revised 9 July 2017 Accepted 14 July 2017
Check for updates

For numbered affiliations see end of article.

Correspondence to

Professor Qian Lin;

13910565673@126.com

\section{ABSTRACT}

Introduction Heart failure with reduced ejection fraction (HFrEF) is defined as the clinical diagnosis of heart failure (HF) and ejection fraction (EF) $\leq 40 \%$, which is a severe public healthcare issue and brings a heavy social and economic burden for patients with HFrEF. Chinese herbal medicine (CHM) has a long history in treating HF. Questions concerning the efficacy and acceptability of CHM-related interventions in adult patients with HFrEF led us to use the method of systematic review and network metaanalysis to integrate direct and indirect evidence to create hierarchies for all CHM.

Methods and analysis Nine medical databases, including PubMed, EMBASE (OVID), the Cochrane Library, Google Scholar, Web of Science, CNKI, VIP, Wanfang Database and CBM will be searched from the date of database inception to June 2015 (updated to March 2017) without language and publication status restriction. Completely randomised controlled trials (RCTs) comparing $\mathrm{CHM}$ or CHM plus routine treatment with $\mathrm{CHM}$, CHM plus routine treatment, routine treatment, no treatment or placebo for adults with HFrEF will be examined. Our primary outcomes will include all-cause mortality, HF-related death, allcause rehospitalisation, HF-related rehospitalisation and acceptability (discontinuation due to any adverse events during treatment). Secondary outcomes will include response rate, mean value or mean difference from baseline of surrogate indexes. We will perform the Bayesian network meta-analyses (NMA) for the most frequently reported primary or secondary outcome and the acceptability outcome, if available. Meta-regression, subgroup analyses and sensitivity analyses will be conducted based on prespecified effect modifiers to assess the robustness of the findings.

Dissemination The results of this NMA will provide useful information about the effectiveness and acceptability of CHM in adults with HFrEF, which will also have implications for clinical practice and further research. Findings will be disseminated through peer-reviewed journal publication and conference presentations.

PROSPERO registration number CRD42016053854.
Strengths and limitations of this study

- As far as we know, this will be the first network meta-analysis to evaluate the effectiveness and acceptability of Chinese herbal medicine in adults with heart failure and reduced ejection fraction. Network meta-analysis based on Bayesian theory can integrate direct evidence and indirect evidence from all alternative treatment options for the same condition to estimate the effect size of all treatments and to rank them.

- Well established eligible criteria, rigorous quality assessment and data collection, standardised statistical analysis, subgroup and sensitivity analyses may increase study strength and reduce heterogeneity.

- The drawbacks of this study may potentially reside in low quality of evidence, publication bias, heterogeneity across studies and inconsistency between direct and indirect evidence, which will decrease the robustness of the results.

\section{BACKGROUND}

Heart failure with reduced ejection fraction (HFrEF) is a clinical syndrome characterised by abnormal systolic function with or without abnormal diastolic function, reduced ejection fraction, and the symptoms and signs of heart failure (HF). According to present guidelines, HFrEF is defined as the clinical diagnosis of $\mathrm{HF}$ and ejection fraction (EF) $\leq 40 \%{ }^{1}$ HF incidence has largely remained stable over the past several decades, with $>650000$ new HF cases diagnosed annually in the United States. ${ }^{1}$ Approximately 9 per 1000 individuals among those aged 35-74 years in China $^{2}$ and 10 per 1000 of the population over 65 years of age in the United States 
have clinically manifest $\mathrm{HF}$, and the prevalence continues to rise. ${ }^{1}$ Being the severe and terminal phase of many cardiovascular diseases, such as hypertension, arrhythmia and coronary heart diseases, HF is a leading cause of death, hospitalisation and rehospitalisation worldwide. ${ }^{3}$ Despite acquired advancements in HF treatments, including guideline-directed medicine therapies (GDMTs), implanted devices and even heart transplantation, still approximately $50 \%$ of people diagnosed with HF will die within 5 years ${ }^{14}$ and the number of deaths with HF was as high in 2011 as it was in $1995,{ }^{4}$ not to mention the high cost of HF-related hospitalisation (about $\$ 23077$ per patient in the United States). ${ }^{5}$ HFrEF makes up about half of the overall HF burden. ${ }^{6}$

In China, Chinese herbal medicine (CHM) has been used to treat symptoms of HF for more than 2500 years, which includes oedema, shortness of breath, dizziness, exercise intolerance, palpitations and so on. The manifestations, pathogeneses and treatments of HF have been discussed and summarised in traditional Chinese medicine (TCM) theory, and CHM used alone or integrated with routine treatments has been widely accepted as an effective method in the treatment of HFrEF in China. In the early 21st century, along with the introduction of evidence-based medical conception and practical methods, many TCM doctors carried out controlled clinical trials to explore the efficacy and safety of diverse CHM on HFrEF, and relevant results were promising. However, various characteristics of these trials yielded different results, which demands further comprehensive evidence. ${ }^{7-9}$ Most trials have focused on comparing CHM alone or CHM plus routine treatments versus placebo or routine treatments, but failed to compare CHM with another CHM. Currently, no published meta-analysis has combined direct and indirect evidence for the use of CHM on HFrEF, though it is important to inform both the physician and the patient which CHM or CHM plus routine treatment may be the most effective and acceptable treatment.

For the above reasons, we will use network meta-analysis (NMA), a methodological approach that allows simultaneous comparison of multiple interventions within a single analysis. Based on the transitivity and similarity between included studies, and preserving randomisation, this NMA will bring improved precision of the estimated effect size and the ability to compare treatments that have not been directly compared in any trial. ${ }^{1011}$ To be specific, this approach will be used to integrate direct evidence (from studies directly comparing interventions) with indirect evidence (from information comparing two interventions via the same comparator) to estimate and rank the effectiveness and acceptability of CHM or CHM plus routine treatment on HFrEF by comparing them with routine treatment, placebo or CHM. ${ }^{12}$
METHODS

Eligible criteria

Types of studies

All peer-reviewed, full-reported prospective parallel randomised controlled trials (RCTs) irrespective of blinding will be assessed, but non-RCTs, small sample studies (less than 20 cases), duplicate reports and pilot studies will be excluded. In addition, the first phase data of randomised crossover trials and cluster RCTs will also be included for analysis.

\section{Types of participants}

Patients diagnosed with HFrEF (New York Heart Association (NYHA) class II or IV) and aged over 18 years old but with no limitations on gender, course and comorbidity will be eligible. Both acute HFrEF and chronic HFrEF will be included, but patients waiting for or having accepted heart transplantation, or suffering heart shock or heart arrest will be excluded. The diagnosis criteria adopted by the included studies should be identical to at least two major criteria or one major criterion in conjunction with two minor criteria of the Framingham standard, ${ }^{13}$ and must fulfil a $\mathrm{EF} \leq 40 \%$ (online supplementary appendix $1)$. For those trials including patients with HFrEF and HFpEF (heart failure with preserved ejection fraction), we will contact the author to obtain data for HFrEF participants. If there is no response, this study will be discarded. Furthermore, to assure transitivity within the network, trials in a single node, for example AC and BC, should be systematically or proportionally similar in critical baseline characteristics, such as gender, age, comorbidity, combined medication and HFrEF severity. ${ }^{14}$

\section{Types of interventions}

The experimental interventions should be combinations of herbs or Chinese proprietary medicines but with a single herb. RCTs comparing CHM or CHM plus routine treatment with $\mathrm{CHM}$, CHM plus routine treatment, routine treatment, no treatment or placebo will be included. Trials comparing the same CHM (sharing the same single herbs) but at different doses and different treatment durations will be treated as one node in the network. We define routine treatment as GDMT (eg, angiotensin converting enzyme inhibitor, angiotensin II receptor blocker, $\beta$ blocker, aldosterone antagonist and hydralazine/nitrate) or inotropic drugs (eg, digaoxin) ${ }^{1}$ which refer to only one GDMT. In addition, we will exclude trials involving a combination of more than two kinds of CHM. Furthermore, distribution with respect to potential effect modifiers (eg, administration route, dose, frequency) among trials in one single node should be similar. ${ }^{14}$

\section{Types of outcome measures}

We will exclude trials with treatment duration of less than 2 weeks. Our primary outcomes will include all-cause mortality, HF-related death, all-cause rehospitalisation, HF-related rehospitalisation and acceptability 
(discontinuation due to any adverse events during treatment). Secondary outcomes will include response rate, mean value or mean difference from baseline of ultrasonic cardiograph indexes (eg, left ventricular ejection fraction (LVEF), left ventricular mass index (LVMI)), NYHA grade, b-type natriuretic peptide (BNP), n-terminal pro-b-type natriuretic peptide (NT-proBNP), blood pressure (BP), weight or quality-of-life score. Only a percentage of improvement of secondary outcomes equal to or more than $50 \%$ will be considered as a response. When a 'response' is not reported, we will use 'remission', if available. Remission is defined as the proportion of patients who do not improve or even worsened..$^{15}$ We will only conduct NMA for two outcomes, including the most frequently reported primary or secondary outcome and the acceptability outcome, if available.

\section{Data sources and search strategy}

A comprehensive search of nine medical databases, including PubMed, EMBASE (OVID), the Cochrane Library, Google Scholar, Web of Science, China National Knowledge Infrastructure (CNKI), Chinese Scientific Journals Database (VIP), Wanfang Database and Chinese Biomedical Database (CBM) will be conducted from the date of database inception to June 2015 (updated to March 2017) without language and publication status restriction. We will search individually or combined mesh with title/abstract terms relating to the intervention of interest (complementary medicine, complementary medicine, oriental medicine, traditional medicine, Chinese medicine, Chinese herbal medicine, Chinese proprietary medicine*, Chinese herbal drug*, Chinese herbal preparation*, herb*, medicinal plant*, plant*, phytomedicine*, botanical) and those relating to the patients of interest (heart failure, cardiac incompensation, cardiac insufficiency, ventricular dysfunction). The above terms in Chinese will be searched in Chinese databases. Also, we will search relevant references of obtained studies and review articles by hand to identify eligible resources. In addition, Chinese Clinical Trial Registry (ChCTR), ClinicalTrials.gov, International Clinical Trials Registry Platform (WHO-ICTRP) will be reviewed for unpublished studies. All relevant authors and manufacturers will be contacted to supplement data for published papers or to provide data for unpublished trials, if available. The search strategy of EMBASE and CENTRAL is available (online supplementary appendix 2).

\section{Study selection}

Four reviewers (JL, YL, JJL and XYC) will independently scan the title and abstract of studies obtained through the search strategy and exclude those failing to meet the eligible criteria. They will then get the full text for potentially relevant trials to further determine whether they fulfil the same eligible criteria. These four reviewers will act as two pairs of reviewers. Reasons for trial exclusion will be documented in detail at full-text level and any disagreement will be resolved by census or consultation with a fifth reviewer (JW).

\section{Data extraction}

Four reviewers (JL, QG, JJL and YCH) will extract data independently using a standardised extraction form. The following items will be extracted: article name, first author or corresponding author, publication year, journal, country, institution and sponsor, trial setting, inclusion criteria, exclusion criteria, details of trial design (ie, randomisation, allocation concealment, blinding), general characteristics of patients (ie, gender, age, HFrEF course and severity, comorbidities), details of intervention and control therapy (ie, components of CHM, CHM type, dosage, intervention duration, co-intervention), sample size, details of outcomes (ie, measuring means, time points, mean value, mean difference, response event, non-response event), and other information that may help detect bias (ie, register ID, data for analysis, termination time). Similarly, division of labour is the same as that in the 'Study selection' section and the fifth reviewer (JW) will be available for consultation regarding controversial issues.

\section{Risk of bias assessment}

The methodological quality of the included studies will be assessed by two reviewers (SNL and JW) using the Cochrane Collaboration's tool, ${ }^{16}$ which is a domain-based evaluation tool to generate a 'Risk of bias' table for each study. The domains for assessment include sequence generation (selection bias), allocation concealment (selection bias), blinding of participants and personnel (performance bias), blinding of outcome assessment (detection bias), incomplete outcome data (attrition bias), selective outcome reporting (reporting bias), and other potential sources of bias (eg, early termination, conflict of interest). As CHM is difficult to blind participants, performance bias will likely be present in all trials. When it comes to objective outcomes such as all-cause mortality, performance bias and detection bias might not be so important so that we can summarise this trial as low risk if bias of other remaining domains is also ranked as low. However, for highly subjective outcomes such as quality-of-life score, we may decide that both blinding of participants and outcome assessment are critical, and high risk of bias for one or more key domains within a study will be evaluated as high risk of bias. In addition, regarding selective outcome, this domain will be ranked as 'low risk' unless the outcomes are critical for our issue, such as all-cause mortality, all-cause rehospitalisation and acceptability. ${ }^{17}$ Also, a third reviewer (KZ) will take charge of any disagreement solution.

\section{Data synthesis and analysis}

First, we will perform pairwise analysis for trials including same experimental intervention using a random-effect model in Stata (version 13.1). Dichotomous data will be calculated as odds ratio (OR) and continuous data as 
weighted mean difference (WMD) or standardised mean difference (SMD), both with corresponding 95\% confidence intervals (CIs). If mean and SD are not provided, we will calculate them from the $\mathrm{p}$ value, $\mathrm{t}$ value, CI or other statistical indices as described in the Cochrane Handbook (version 5.1.0) ${ }^{17}$ Heterogeneity across trials of each pairwise comparison will be assessed by the Cochran $Q$ test and presented as $\mathrm{I}^{2}$ statistics. ${ }^{17}$ Funnel plot and Egger's test will be performed to detect publication bias, if at least 10 studies are available. ${ }^{17}$

Second, we will do NMA to combine all direct and indirect evidence to compare the relative effect size of different CHM treatments, CHM plus routine treatment, routine treatment, placebo, no treatment with each other from the median of the posterior distribution. ${ }^{10} 12$ OR, SMD or WMD will be calculated with corresponding $95 \%$ credible interval $(\mathrm{CrI})^{10}$ and reported in a league table. We will fit the model within a Bayesian framework by using WinBUGS (version 1.4.3) or GeMTC (version 0.14.3) software to perform random-effect multitreatment meta-analysis and Stata or R (version 3.3.0) software to plot and analyse further.

To pool estimate effects, two Markov chains will be run simultaneously by choosing different initial values. Trace plots and the Brooks-Gelman-Rubin statistic will be generated to assess convergence. ${ }^{18}$ To ensure convergence, the previous 50000 samples will be abandoned and described as 'burn in', and posterior summaries will be based on 100000 subsequent simulations. To assess global heterogeneity in the network, we will calculate the $\mathrm{I}^{2}$ statistic using the GeMTC R package (version 3.2.2), which is based on the magnitude of the heterogeneity variance parameter estimated from the NMA models. ${ }^{19}$ To measure inconsistency between direct and indirect evidence, three methods will be used, including the node-splitting method (by separating evidence of one particular comparison into direct and indirect evidence to find inconsistency between them), ${ }^{20}$ the local (loop-specific approach, evaluating inconsistency in each closed loop by generating an inconsistency factor and CI shown in a forest plot) and global (by using the 'designby-treatment' model to compare the difference between consistency and inconsistency models based on a $\chi^{2}$ test) method. ${ }^{21}$ All these methods will serve to produce a more robust conclusion. If there are controversial results among different methods, we will make a comprehensive conclusion based on the ratio of results, heterogeneity and overall risk of bias. To provide a hierarchy of interventions, ranking probability will be calculated and shown as a surface under the cumulative ranking curve (SUCRA) and rankograms. ${ }^{12}$ Also, to detect publication bias in each NMA, comparison-adjusted funnel plots will be drawn, if enough trials are available. ${ }^{22}$

In addition, to eliminate or explore sources of heterogeneity or inconsistency, meta-regression, subgroup or sensitivity analysis for primary outcome will be performed based on prespecified effect modifiers as follows: year of publication, study quality, trial publishing status, sample size, NYHA grade, EF value, comorbidity, age, gender, CHM type, treatment duration, etc. Furthermore, we will use a grade framework to assess the quality of evidence attributed to each primary outcome in one network, with quality-assessment domains including study limitations, imprecision, inconsistency, indirectness and publication bias. ${ }^{23}$

\section{Ethics and dissemination}

As no individual patient data will be used in this NMA, it is not necessary to declare ethics here. The protocol of this NMA has been drawn up in compliance with items recommended by the PRISMA Extension Statement for Reporting of Systematic Reviews Incorporating Network Meta-analyses of Healthcare Interventions ${ }^{24}$ and registered at PROSPERO with the number-CRD42016053854 (available from https://www.crd.york.ac.uk/PROSPERO/display_record.asp?ID=CRD42016053854).

The results of this NMA will provide a more comprehensive and more reliable overview of efficacy and acceptability of CHM for adults with HFrEF by drawing on available direct and indirect evidence and a series of critical appraisal procedures, which will also have implications for clinical practice and further research. Once completed, the findings of this NMA will be published in a peer-reviewed journal and presented at conferences.

\section{Author affiliations}

${ }^{1}$ Beijing University of Chinese Medicine, BeiJing, China

${ }^{2}$ Cardiovascular Department, Dongfang Hospital, Beijing University of Chinese Medicine, Beijing, China

${ }^{3}$ Department of Scientific Research, Dongfang Hospital, Beijing University of Chinese Medicine, Beijing, China

${ }^{4}$ Intensive Care Unit, Dongfang Hospital, Beijing University of Chinese Medicine, Beijing, China

${ }^{5}$ Beijing Institute of Traditional Chinese Medicine, Beijing Hospital of Traditional Chinese Medicine, Capital Medical University, Beijing, China

${ }^{6}$ Department of Education, Dongfang Hospital, Beijing University of Chinese Medicine, Beijing, China

Contributors $Q L$ and $J L$ conceived the NMA. $J L$ and $J J L$ drafted and revised this protocol. JL, JJL, JW, YL, XYC, QG, YCH and SNL established the Data Management Committee and are separately responsible for study selection, data extraction and risk of bias assessment procedures. JW and KZ took charge in coordinating every piece of work together, disagreement settlement and data safety. QL and $\mathrm{JL}$ contributed to the design of statistical analysis. JL and JJL are responsible for overall data synthesis and analysis. QZD contributed to language polishing. QL is responsible for the overall quality of the NMA. All authors read this manuscript and approved the publication of this protocol.

Funding This study is supported by grants from Project of National Natural Science Foundation of China. (No. 81373779).

Competing interests None declared.

Provenance and peer review Not commissioned; externally peer reviewed.

Open access This is an open access article distributed in accordance with the Creative Commons Attribution Non Commercial (CC BY-NC 4.0) license, which permits others to distribute, remix, adapt, build upon this work non-commercially, and license their derivative works on different terms, provided the original work is properly cited and the use is non-commercial. See: http://creativecommons.org/ licenses/by-nc/4.0/

(C) Article author(s) (or their employer(s) unless otherwise stated in the text of the article) 2018. All rights reserved. No commercial use is permitted unless otherwise expressly granted. 


\section{REFERENCES}

1. Yancy CW, Jessup M, Bozkurt B, et al. ACCF/AHA guideline for the management of heart failure: a report of the American College of Cardiology Foundation/American Heart Association Task Force on Practice Guidelines. Circulation 2013;2013:e240- e327.

2. Gu D-feng, Huang Y-guang, $\mathrm{He} \mathrm{J}$, et al. Epidemiological survey on the prevalence of heart failure in China-a multicenter cooperative $\operatorname{study}(A)$. Beijing, China(C): The Seventh National Conference on cardiovascular diseases of Chinese Medical Association, 2002.

3. Berry C, Murdoch DR, McMurray JJ. Economics of chronic heart failure. Eur J Heart Fail 2001;3:283-91.

4. Mozaffarian D, Benjamin EJ, Go AS, et al. Heart disease and stroke statistics-2015 update: a report from the American Heart Association. Circulation 2015;131:e29-e322.

5. Wang G, Zhang Z, Ayala C, et al. Costs of heart failure-related hospitalizations in patients aged 18 to 64 years. Am J Manag Care 2010;16:769-76.

6. Owan TE, Redfield MM. Epidemiology of diastolic heart failure. Prog Cardiovasc Dis 2005;47:320-32.

7. Xu G, Wang Q, Liu K, et al. Clinical trials of qiliqiangxin capsule in the treatment of chronic congestive heart failure. Chin J Diffic and Complic Cas 2008;7:262-4.

8. Yang Z, Deng Y. Observation the effect of qiliqiangxin capsule on heart failure with reduced ejection fraction. Chin Fore Med Treat 2010;5:113.

9. Li X, Zhou HOngmei, Hu Y. Observation the effect of qiliqiangxin decoction on 70 patients diagnosed as heart failure with reduced ejection fraction. 2010;32:71-2.

10. Salanti G, Higgins JP, Ades AE, et al. Evaluation of networks of randomized trials. Stat Methods Med Res 2008;17:279-301.

11. Caldwell DM, Ades AE, Higgins JP. Simultaneous comparison of multiple treatments: combining direct and indirect evidence. BMJ 2005;331:897-900.

12. Lu G, Ades AE. Combination of direct and indirect evidence in mixed treatment comparisons. Stat Med 2004;23:3105-24.
13. McKee PA, Castelli WP, McNamara PM, et al. The natural history of congestive heart failure: the framingham study. $N$ Engl $J$ Med 1971;285:1441-6.

14. Salanti G. Indirect and mixed-treatment comparison, network, or multiple-treatments meta-analysis: many names, many benefits, many concerns for the next generation evidence synthesis tool. Res Synth Methods 2012;3:80-97.

15. Wen-Ting S, Fa-Feng C, Li X, et al. Chinese medicine shenfu injection for heart failure: a systematic review and meta-analysis. Evid Based Complement Alternat Med 2012;2012:1-25.

16. Higgins JP, Altman DG, Gøtzsche PC, et al. Cochrane bias methods group; cochrane statistical methods group. The cochrane collaboration's tool for assessing risk of bias in randomized trials. BMJ 2011;343:d5928.

17. Higgins JPT, Green S. Cochrane handbook for systematic reviews of interventions version 5.1. 0. The Cochrane Collaboration, 2011. updated March 2011.

18 Brooks SP, Gelman A. General methods for monitoring convergence of iterative simulations. J Computa and Grap Stat 1998;7:434-55.

19 Neupane B, Richer D, Bonner AJ, et al. Network meta-analysis using $\mathrm{R}$ : a review of currently available automated packages. PLoS One 2014;9:e115065.

20 Dias S, Welton NJ, Caldwell DM, et al. Checking consistency in mixed treatment comparison meta-analysis. Stat Med 2010;29:932-44.

21 Chaimani A, Higgins JP, Mavridis D, et al. Graphical tools for network meta-analysis in STATA. PLoS One 2013;8:e76654.

22 Salanti G, Ades AE, loannidis JP. Graphical methods and numerical summaries for presenting results from multiple-treatment metaanalysis: an overview and tutorial. J Clin Epidemiol 2011;64:163-71.

23 Salanti G, Del Giovane C, Chaimani A, et al. Evaluating the quality of evidence from a network meta-analysis. PLoS One 2014;9:e99682.

24 Hutton B, Salanti G, Caldwell DM, et al. The PRISMA extension statement for reporting of systematic reviews incorporating network meta-analyses of health care interventions: checklist and explanations. Ann Intern Med 2015;162:777380-497. 